\title{
Early Markers of Angiogenesis and Ischemia during Bowel Conduit Neovascularization
}

\author{
Oscar J. Manrique, $\mathrm{MD}^{1}$ Pedro Ciudad, MD, $\mathrm{PhD}^{2} \quad$ Alex Wong, $\mathrm{MD}^{3}$ Federico Lo Torto, $\mathrm{MD}^{2}$ \\ Yun-Fen Li, MD ${ }^{4}$ Pei-Yu Chen, MD ${ }^{5}$ Doan-Minh Nguyen, MD, PhD ${ }^{1}$ Hung-Chi Chen, MD, PhD ${ }^{2}$
}

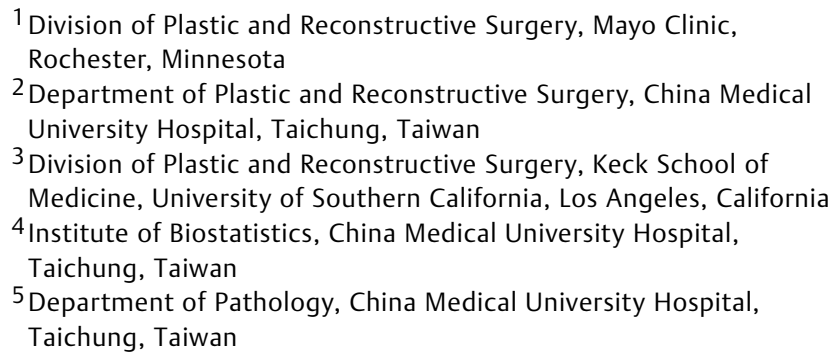

Address for correspondence Oscar J. Manrique, MD, Division of Plastic and Reconstructive Surgery, Mayo Clinic, 200 First Street SW, Rochester, MN 55905 (e-mail: manrique.oscar@mayo.edu).

J Reconstr Microsurg

\begin{abstract}
Keywords

- microsurgery

- bowel conduits

- neovascularization

Background Bowel flaps are a good and reliable method to restore the continuity of the aerodigestive tract. Radiated fields, contaminated recipient sites, or depleted recipient vessels may increase the risk for ischemic injury after transfer. During ischemic events, we believe that bowel conduits with serosa have a delayed neovascularization process at its new recipient site.

We conducted an ischemia/reperfusion murine model to understand the difference among bowel conduits with and without serosa.

Materials and Methods Two groups of rats were compared: control group (jejunal conduit with serosa) and a target group (jejunal conduit without serosa). These conduits were harvested from the peritoneal cavity and transferred into a subcutaneous pocket. After 72 hours of transfer and pedicle ligation, histological changes related to ischemia/reperfusion were assessed. In addition, tissue markers of angiogenesis (CD34), ischemia (lactate dehydrogenase [LDH]), and inflammation (interleukin [IL]-1 $\beta$ and IL-6) were analyzed.

Results Two groups $(n=20)$ of male rats were analyzed. Histology showed intact jejunal mucosa in the target group. The control group showed decreased number of mucin, globet cells, decreased height, and fragmentation of villi with the absence of intestinal glands. Markers of angiogenesis (CD34) were higher in the target group. In addition, markers of ischemia $(\mathrm{LDH})(p=0.0045)$ and inflammation (IL-1b, $p=0.0008$, and IL-6, $p=0.0008$ ) were significantly lower in the target group as compared with the control group.

Conclusions In circumstances in which the recipient site does not offer an adequate and healthy bed or a vascular insult occurs, bowel flaps with less amount of serosa may be able to neovascularize faster thereby increasing its chances of survival.
\end{abstract}

received

February 10, 2017

accepted after revision

May 10, 2017
Copyright $\odot$ by Thieme Medical

Publishers, Inc., 333 Seventh Avenue, New York, NY 10001, USA.

Tel: +1(212) 584-4662.
DOI https://doi.org/

10.1055/s-0037-1603905. ISSN $0743-684 \mathrm{X}$. 
Reconstruction of the pharyngoesophageal tract with bowel flaps has been described as a good and reliable method to restore the continuity of the aerodigestive tract. ${ }^{1-5}$ However, when clinical factors such as a radiated field, contaminated recipient site or depleted recipient vessels are encountered, an alternative surgical approach is necessary. With any ischemic insult, bowel mucosa becomes very sensitive to prolonged absence of vascular supply or congestion, leading to epithelial damage, bacteria translocation, and possible failure of the conduit. ${ }^{6}$ In an effort to find ways to limit bowel ischemia following transfer, animal models using prefabricated bowel flaps have been described. ${ }^{7}$ Several of these studies have evaluated ways to promote early and faster neovascularization of the transferred bowel segment to prevent stricture, limit epithelial damage, and prevent perforation. One of the main anatomical differences between some common flaps used to reconstruct the aerodigestive track, such as the jejunum or the ileocolon flaps, is the lack of serosa in the posterior wall of the ileum and colon. ${ }^{8,9}$

Based on our clinical experience, we hypothesize that the serosa may delay the neovascularization process after the bowel conduit has been transferred to its recipient site.

Using a validated murine model, ${ }^{7}$ the purpose of this study was to examine the physiological response of bowel conduits with and without serosa to analyze the neovascularization and integration process of these conduits after transfer under simulated ischemia.

\section{Material and Methods}

\section{Animal Model}

Male Sprague Dawley rats (BioLASCO, Taiwan Co.) weighing between 350 and $500 \mathrm{~g}$ were used under the animal care and laboratory standards in accordance with the guidelines of China Medical University Hospital. Two groups were compared: group A (control), jejunal conduit with intact serosa, and group $B$, jejunal conduit with no serosa. The animals were anesthetized with an intramuscular injection of Zoletil (tiletamine $40 \mathrm{mg} / \mathrm{kg}$; Virbac, Australia). In addition, preoperative antibiotics (gentamicin $4 \mathrm{mg} / 0.2 \mathrm{~mL}$ ) were administrated intramuscularly before surgery. The abdominal skin was shaved and prepared with povidone-iodine. Through midline laparotomy (-Fig. 1), a 4-cm segment of jejunum was visualized and divided keeping the vascular pedicle straight to prevent twisting. The chosen segment was based on a uniform blood vessel distribution to the bowel conduit. In addition, we preserved the anatomical peristaltic direction of the bowel conduit prior to its transfer into an abdominal subcutaneous pocket to prevent twisting of the bowel (-Figs. 2 and 3). After the segment of bowel was divided and under microscope magnification $(40 \times$, Leica Microsystems, model M651, Schweiz AG), the serosa was removed sharply using curved microsurgical scissors. The average time used to remove the serosa from each bowel segment was 40 minutes (range: $35-45$ minutes). After the bowel segment was harvested, the proximal and distal ends of the rest of the bowel were anastomosed using 6-0 nylon, thus restoring the continuity of the gastrointestinal tract.

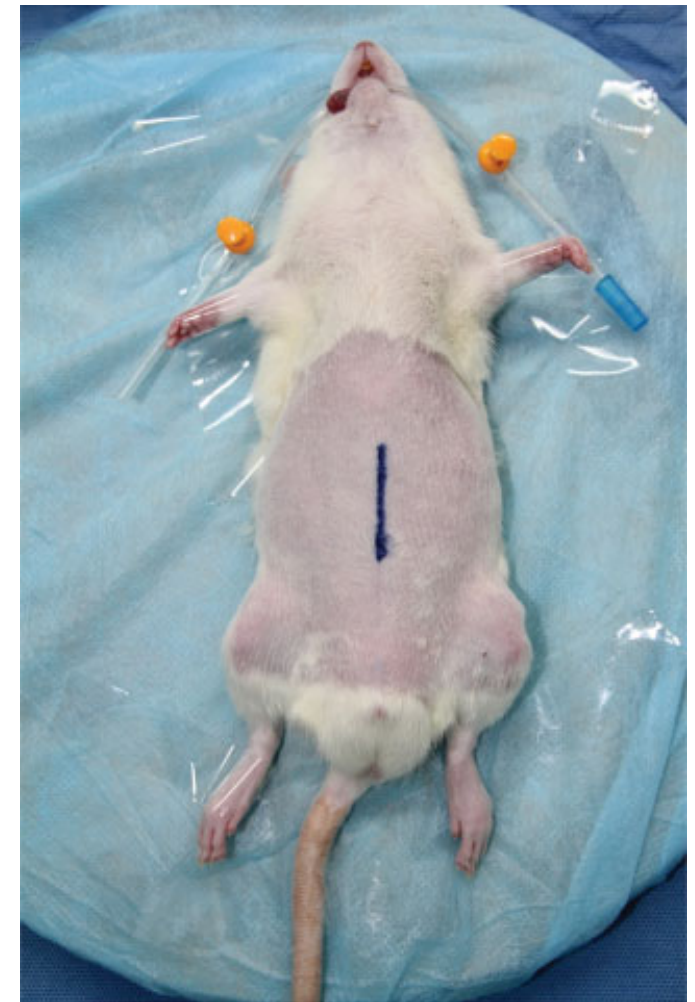

Fig. 1 Under anesthesia, the animal's jejunum is accessed through a midline laparotomy incision.

While still attached to its pedicle, the segment of jejunum was mobilized and brought out of the abdominal cavity and placed under a subcutaneous skin pocket. A 6-0 nylon loop was placed at the base of the vascular pedicle to ligate it 72 hours after transfer without disrupting the possible new areas of neovascularization between the conduit and surrounding tissues. The midline laparotomy was closed with interrupted 4-0 nylon, leaving a small hiatus to allow free passage of the jejunal vascular pedicle (-Fig. 3 ). The bowel conduit was irrigated and a distal jejunostomy was made on the left side of the abdominal skin to prevent accumulation of secretions in the postoperative period and to monitor the perfusion of the bowel conduit. After the vascular pedicle was ligated, the jejunal conduit was maintained under the skin flap for additional 72 hours. At that point, the jejunal conduit was harvested for further tissue and blood analysis. For group B, the same procedures were performed, but in addition, prior to placing the jejunal conduit in the subcutaneous pocket, careful serosal stripping was performed under the microscope as previously described. Viability of the bowel was assessed by gross appearance (color and peristalsis), and histological examination was performed 72 hours after pedicle ligation. Histological sections were prepared with hematoxylin and eosin stains and viewed under $100 \times$ and $200 \times$ magnification looking for ischemic changes in the bowel epithelium. In addition, markers of bowel angiogenesis, such as CD34, bowel ischemia, such as lactate dehydrogenase (LDH), and bowel inflammation, such as IL- $1 \beta$ and IL-6, were recorded. To compare the difference 


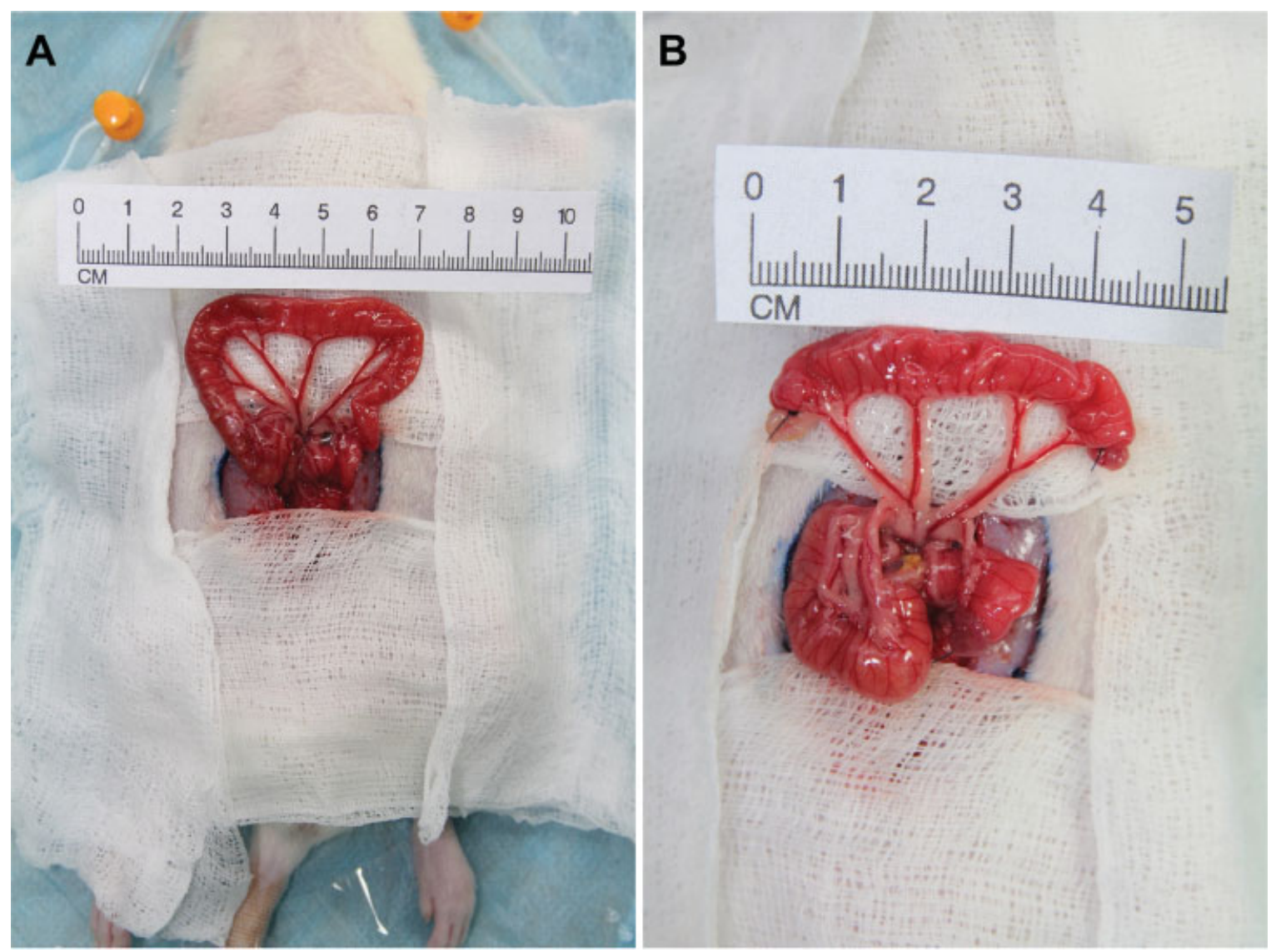

Fig. 2 (A) Exploration of jejunum with complete mesenteric arcade prior to division. (B) Division of proximal and distal jejunum ends with intact mesenteric vascular supply.

among our groups, a Wilcoxon rank sum-test was performed. Statistical analysis was conducted using SAS 9.4 software (SAS Institute, Cary, NC). All statistical tests were two-sided with a significance level of 0.05 .

\section{Results}

After 72 hours of ligation, 20 jejunal conduits were harvested ( $n=10$ serosa; $n=10$ no serosa). Histological sections using hematoxylin and eosin stains of the jejunum were compared under $100 \times$ and $200 \times$ magnification. Our control group (bowel with serosa) showed decrease number of mucin, globet cells, decrease height, and fragmentation of villous with the absence of intestinal glands. However, in our stripped serosal model, they showed intact jejunal mucosa with tall intestinal villi, deep intestinal glands (crypts of Lieberkuhn), and numerous plump globet cells (-Fig. 4).

To correlate our previous findings and to look for other signs of angiogenesis, CD34 stains were also performed. Histological sections at $200 \times$ magnification showing CD34 immunohistochemistry stain of bowel mucosa 72 hours after pedicle ligation were analyzed. A scattered number of CD34 with fibrosis and decreased amount of capillaries at the endothelium level were observed in our control group. However, an increased number of CD34 stains over bowel mucosa (black arrows) with increased number of capillaries were seen in the rats with serosal stripping (-Fig. 5).

Looking for biomarkers of ischemia, LDH was obtained in blood samples of both groups 72 hours after ligation. Comparing LDH levels among our control and experimental group, higher levels of LDH were seen in our control group (median 952.5 vs. $625.5 \mathrm{IU} / \mathrm{L} ; p=0.0045$; - Fig. 6).

In addition, biomarkers of inflammation such as IL- $1 \beta$ and IL-6 were compared among groups. After 72 hours of ligation, tissue samples were obtained. IL- $1 \beta$ and IL- 6 were significantly higher in the control group as compared with the no-serosa model (median 679.5 vs. $276.5 \mathrm{pg} / \mathrm{mL}$, $p=0.0008$ for IL-1 $\beta$ [-Fig. 7]; median $67.5 \mathrm{pg} / \mathrm{L}$ vs. $15.0 \mathrm{pg} / \mathrm{L}, \boldsymbol{p}=0.0008$ for IL-6 [-Fig. 8]).

\section{Discussion}

In 1992, Kawahara et al described the first experience of bowel conduits for pharyngolaryngectomy reconstruction. ${ }^{10}$ In addition, several other studies have shown the efficacy, reliability, and promising results used in aerodigestive tract reconstruction with minimal donor-site morbidity.,6,11-15 However, due to the low threshold for ischemia, interruption in its vascular supply will result in hypoxia and injury to the intestinal epithelium. At the same time, the inflammation generated by this vascular event will increase the oxygen 


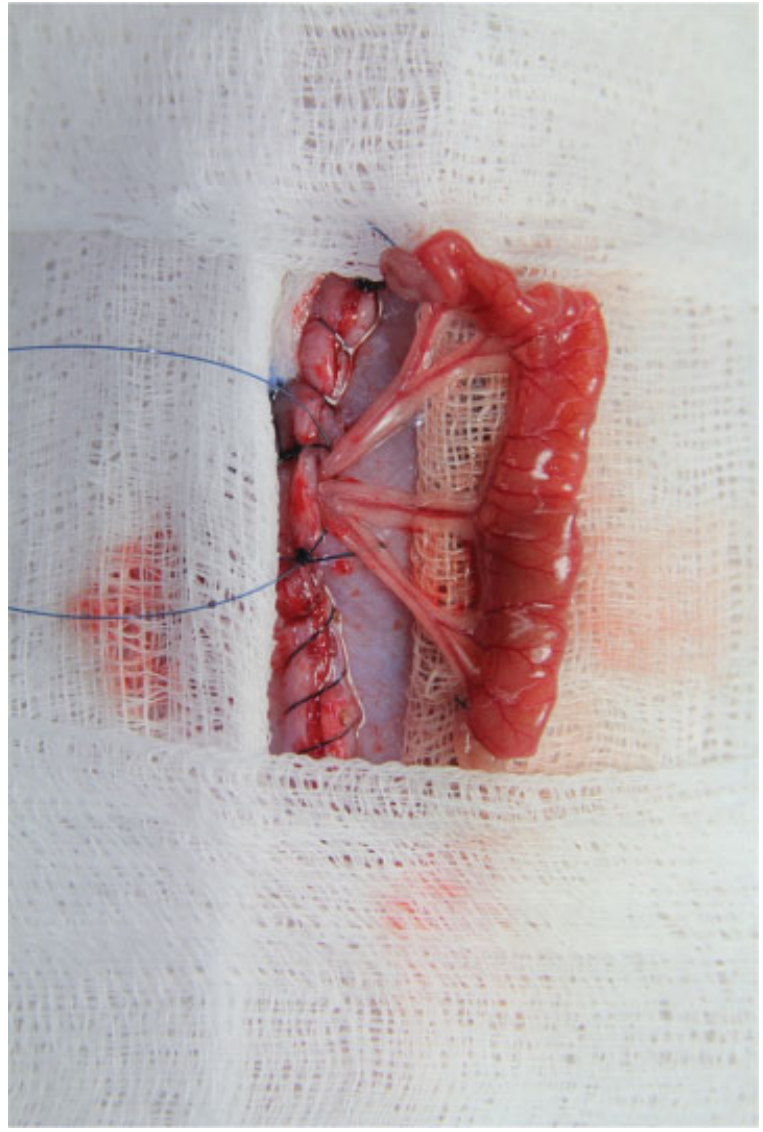

Fig. 3 Closure of midline laparotomy leaving a small hiatus for passage of the mesenteric pedicle supplying the segment of the jejunum. A loop of 6-0 nylon is left at the base of the pedicle for ligation 72 hours after placement in the subcutaneous pocket.

consumption of the affected cells. ${ }^{16}$ This is due to their large surface area in conjunction with their complex vascular supply system. ${ }^{17}$ In addition, the anaerobic environment of the bowel lumen will lead to an extremely steep oxygen gradient across the bowel epithelium, leading to bacterial translocation and possible sepsis. ${ }^{16}$
Based on our clinical experience, pharyngolaryngectomy reconstruction in patients who lack recipient site vessels, history of recipient site radiation, or heavy contamination can become very challenging. ${ }^{6}$ When these issues are anticipated, selection of an appropriate bowel flap is important. Conduits with a quicker process of neovascularization and that integrates faster into its recipient site are preferred. One of the most common type of bowel flaps used during pharyngolaryngectomy reconstruction are the jejunum and the ileocolon flaps. ${ }^{5}$ In the last decade, our group has conducted a significant number of aerodigestive track reconstructions using free and pedicle bowel flaps. $8,12,13,18-20$

Even though the success rate of these flaps is high, ${ }^{6}$ we have noticed that when a ischemic event occurs, the portion of the bowel conduit that survives is usually the one not covered by serosa (e.g., posterior wall of the terminal ileum and ascending colon). However, and up to now, no study has demonstrated this physiological process among bowel conduits with and without serosa during ischemic events.

To analyze and understand the different mechanisms involved in bowel ischemia/reperfusion injury, inflammation, and angiogenesis, we decided to compare among our groups different biological markers involved in this process.

In bowel ischemia, LDH is an important marker that correlates with the index of cell injury and bowel ischemia/reperfusion. ${ }^{21-24}$ The reduction of oxygen supply impairs the mitochondria respiration process and triggers a cytoplasmic low-performance, energy-producing process. This will eventually turn from an aerobic to an anaerobic metabolism, having lactate as the end product of this pathway. The low LDH levels $(p=0.0045)$ seen in our stripping serosa model most likely represent a reduced ischemic insult due to a faster integration process and angiogenesis at the recipient site (-Fig. $\mathbf{6}$ ).

The low LDH levels also correlate with the histopathological findings seen in the same group, a more intact histological architecture as seen on hematoxylin and eosin examination 72 hours after pedicle ligation ( - Fig. 4). The intact jejunal mucosa with tall intestinal villi, deep intestinal
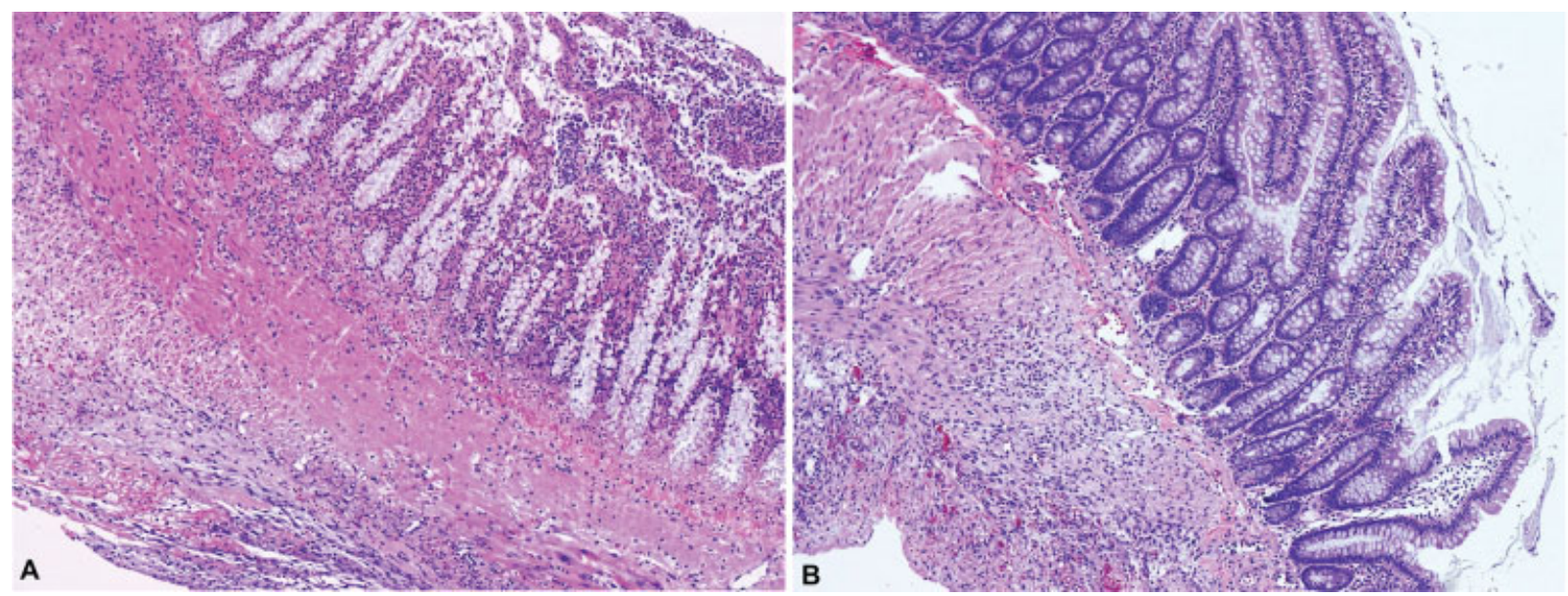

Fig. 4 Histological section (hematoxylin and eosin stain) at 100× magnification showing bowel mucosa 72 hours after pedicle ligation. (A) The control group shows decreased number of mucin, globet cells, decreased height, and fragmentation of villous with the absence of intestinal glands. (B) Intact jejunal mucosa with tall intestinal villi, deep intestinal glands (crypts of Lieberkuhn), and numerous plump globet cells. 


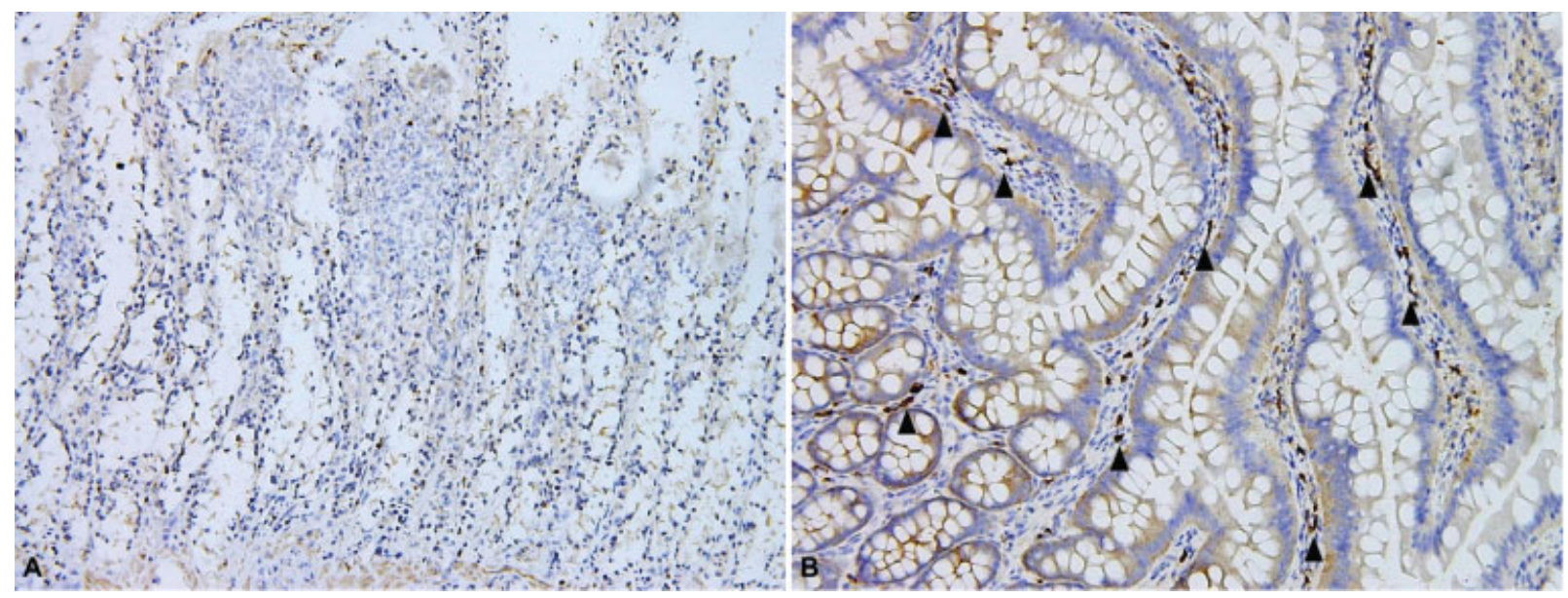

Fig. 5 Histological section at $200 \times$ magnification showing CD34 immunohistochemistry stain of bowel mucosa 72 hours after pedicle ligation. (A) Scattered number of CD34 with fibrosis and decreased amount of capillaries at the endothelium level in the control group (rats with complete serosa). (B) Increased number of CD34 stains over bowel mucosa (black arrows) with increased number of capillaries in rats with serosal stripping.

glands (crypts of Lieberkuhn), and numerous plump globet cells confirms a possible decreased ischemic event with a faster angiogenesis phase.

Since the late 1990 s, CD-34 has been described as a strong marker for endothelial cell proliferation, vascular regeneration, angiogenesis, and tissue healing. ${ }^{25-27}$ In ischemic events, progenitor cells mobilize and adhere to sites of vascular lesions inducing endothelial proliferation. In our jejunal conduit model, the group lacking of serosa demonstrated an increase in the number of CD-34 cells, as seen in - Fig. 5. The increased number of vessels represents a stronger neovascularization and angiogenesis process of the deserosalized conduit at the recipient site. This physiological response might allow the transferred conduit to integrate faster and survive in the event of occlusion of its main pedicle based on its new blood supply, as demonstrated in our murine model.

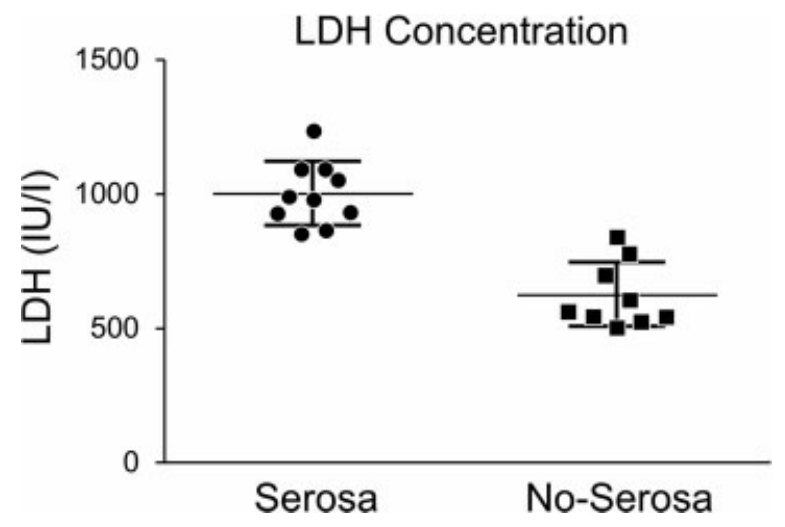

Fig. 6 Lactate dehydrogenase in blood samples for the serosa and the no-serosa groups (median $952.5 \mathrm{IU} / \mathrm{L}$ for the serosa group vs. $625.5 \mathrm{IU} / \mathrm{L}$ for the no-serosa group; $p=0.0045$ ). The three horizontal lines are the third quartile, median, and the first quartile, respectively, from top to bottom.

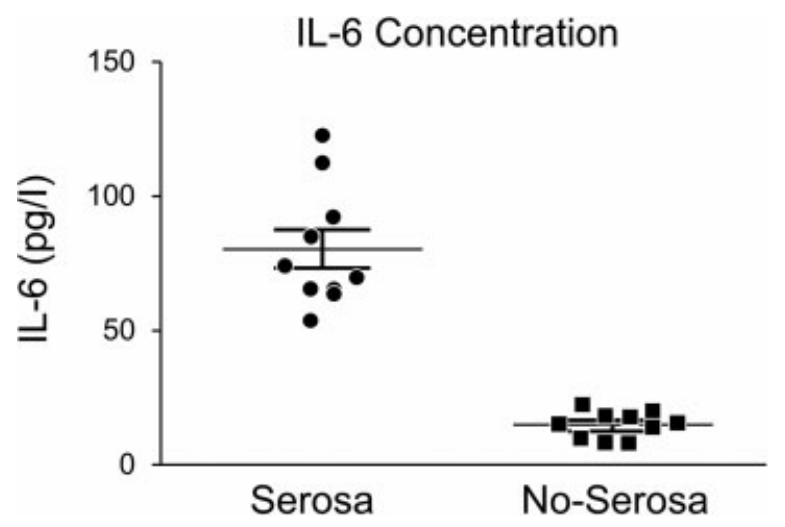

Fig. 7 Interleukin-1 $\beta$ compared between the groups of serosa and no-serosa (median 679.5 vs. $276.5 \mathrm{pg} / \mathrm{mL} ; p=0.0008$ ). The three horizontal lines are the third quartile, median, and the first quartile, respectively, from top to bottom.

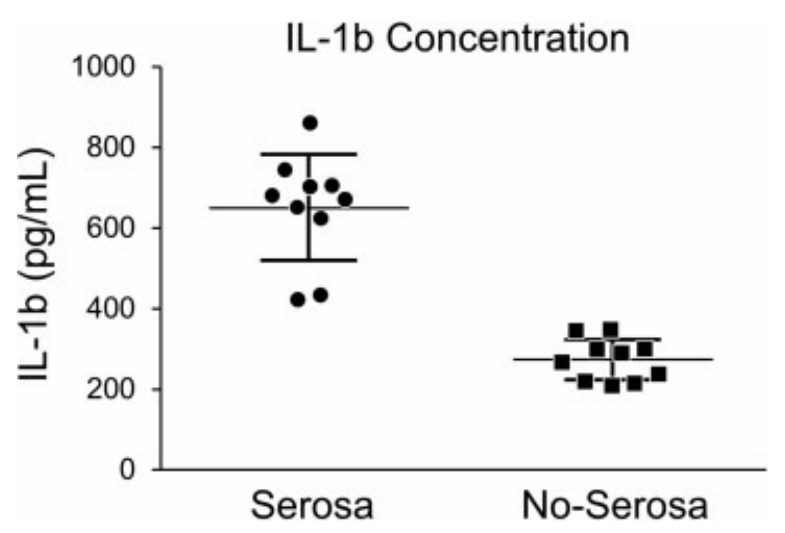

Fig. 8 Interleukin-6 compared between the groups of serosa versus no-serosa (median 67.5 vs. $15.0 \mathrm{pg} / \mathrm{L} ; p=0.0008$ ). The three horizontal lines are the third quartile, median, and the first quartile, respectively, from top to bottom. 
During an ischemic injury insult, several other proinflammatory cytokines such as IL- $1 \beta$ and IL- 6 have been shown to be important markers of tissue injury. ${ }^{28}$ The role of these interleukins is to lead to the influx and activation of neutrophils, resulting in inflammation of the injured tissue. Both of these cytokines are potent pleiotropic, proinflammatory mediators responsible for the acute-phase response during ischemic events.

The low levels of both cytokines (IL-1 $\beta$ and IL-6) encountered in our deserosalized animal model also shows a lesser degree of inflammation and ischemic injury. This might represent a faster adaptation and angiogenesis process of the conduit into its new recipient site ( - Figs. $\mathbf{7}$ and $\mathbf{8}$ ).

The serosa represents the most external layer of the bowel. This layer consists of connective tissue covered by a simple squamous epithelium, which reduces frictional forces during digestive movements by secreting serous fluid. The serosal layer forms a natural barrier from the spread of inflammatory and other malignant processes.

Free tissue transfer based on bowel conduits for pharyngolaryngectomy reconstruction has been used for many years with very promising outcomes. ${ }^{10,14,15,19}$ From the clinical standpoint and based on many years of clinical observation by the senior author, $3,5,8,11,12,18,19$ the physiological response of some of these bowel conduits during ischemic events is different. The lack of serosa in the posterior aspect of the ileum and colon compared with an intact serosal layer throughout the circumference of the jejunum is a possible hypothesis for the difference in behavior of these conduits during a period of ischemic/reperfusion injury. However, up to this point and to our knowledge, no prior study has demonstrated this difference. Using a murine model of ischemia/reperfusion injury, we compared the same segment of bowel with and without serosa to prove our theory.

This technique could be extrapolated to humans; however, there could be several challenges and limitations such as an increased operating time since the bowel conduit required for a normal esophageal reconstruction will be longer, and this technique has also a surgical learning curve. In addition, the patient will be exposed to the potential risks of this technique such as full thickness injuries, possible bowel leaks, necrosis, and compromise of the blood supply to the conduit.

The main purpose of using this technique was to create a murine model that mimics the normal lack of serosa in the posterior wall of the terminal ileum and ascending colon and compare this to the complete lining of serosa in the jejunum.

We believe that one alternative is to strip the serosa of the mesentery, as this will avoid the potential complications related to performing this technique over bowel and will save operating time.

However, and up to now, there are no other techniques or alternatives that describe a complete deserosalization of the bowel, as we believe this technique is too risky to extrapolate in humans.

Based on defined markers of ischemia, inflammation, and angiogenesis, we were able to find some of the answers to

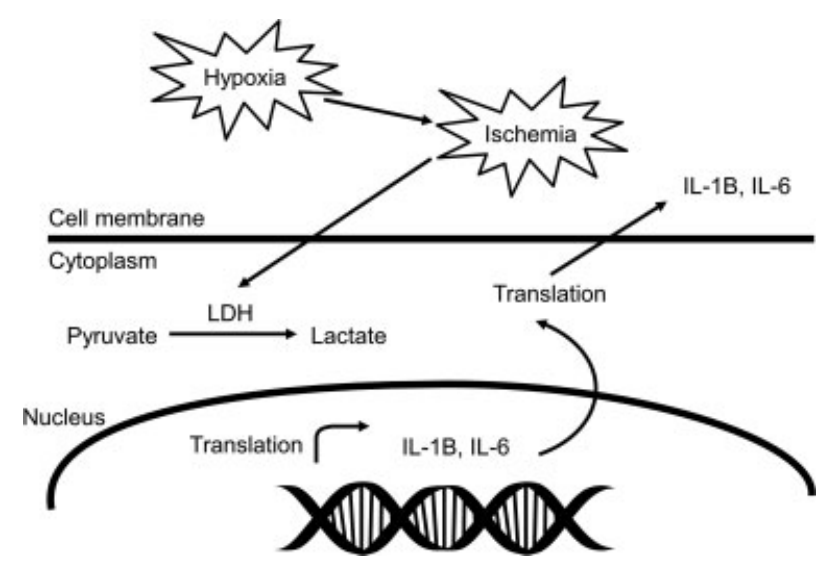

Fig. 9 Ischemic bowel injury mechanism. An ischemic event will lead to an anaerobic metabolism, catalyzing pyruvate through lactate dehydrogenase into lactate. In addition, at the nuclear level, there is an activation of proinflammatory cytokines such as interleukin (IL)-1 $\beta$ and IL-6.

our question. The segment of bowel that lacked serosa was able to survive and to show a lesser degree of ischemic injury. On gross appearance, the color and motility were better as compared with the conduit with an intact serosal layer. At the molecular level, the increased number of CD34 cells with a better histological pattern demonstrates a lesser degree of ischemia with a faster angiogenesis phase at its recipient site. In addition, the decreased levels of LDH, IL-1 $\beta$ and IL-6 correlate with a decreased level of inflammation and a better adaptation/neovascularization process of the free bowel conduit ( - Fig. 9). Extrapolating these data into the clinical setting, we can now presume why during an ischemic insult some of these bowel conduits have a different physiological behavior. Based on these results, we believe that the process of angiogenesis and neovascularization of these bowel conduits is different during ischemic insults, making the bowel flaps with less serosa a faster conduit to adapt and neovascularize.

\section{Conclusions}

The use of free bowel conduits has been shown to be a safe and effective treatment option. However, based on the patient's clinical background, appropriate selection of a bowel conduit is imperative to minimize complications during possible ischemic events.

\section{Note}

This paper was presented at the American Society of Reconstructive Microsurgery, Waikoloa, HI, 2017.

\section{References}

1 Sacak B, Orfaniotis G, Nicoli F, et al. Back-up procedures following complicated gastric pull-up procedure for esophageal reconstruction: salvage with intestinal flaps. Microsurgery 2016;36(07): 567-572 
2 Orfaniotis G, Sacak B, Nicoli F, et al. Absence of the ileocolic artery: microsurgical implications in reconstruction of the esophagus with the free ileocolon flap. Microsurgery 2016;36(02):173-174

3 Imaizumi A, Liem AA, Yang CF, Chen W, Chen SH, Chen HC. Longterm outcomes of simultaneous skin and bowel flaps for esophageal reconstruction. Ann Plast Surg 2015;75(02):180-185

4 Perrone F, Nitto A, Tang YB, Chen SH, Chen HC. Three uses of an extra length of jejunum in pharyngo-oesophageal reconstruction with free jejunal flap. J Plast Reconstr Aesthet Surg 2013;66(01): 16-22

5 Chen HC, Kim Evans KF, Salgado CJ, Mardini S. Methods of voice reconstruction. Semin Plast Surg 2010;24(02):227-232

6 Perrone F, Gharb BB, Rampazzo A, Ngo QD, Chen SH, Chen HC. Evaluation and management of complications or functional problems at the recipient site after esophageal and voice reconstruction with free ileocolon flap. Surgery 2013;153(03):373-382.e2

7 Tan BK, Chen HC, Wei FC, et al. Formation of independently revascularized bowel segments using the rectus abdominis muscle flap: a rat model for jejunal prefabrication. Plast Reconstr Surg 2002;109(02):655-663

8 Gharb BB, Rampazzo A, Hsu CT, Bassetto F, Spanio di Spilimbergo $\mathrm{S}$, Chen HC. Histological changes of the voice tube and possible clinical consequences after voice reconstruction with free ileocolon flap. Ann Plast Surg 2013;71(01):109-113

9 Perrone F, Gharb BB, Rampazzo A, Ngo QD, Chen HC. The use of a chimaeric sero-muscular flap to prevent anastomotic leakage in head and neck reconstruction with free ileocolon flap. J Plast Reconstr Aesthet Surg 2012;65(06):752-756

10 Kawahara H, Shiraishi T, Yasugawa H, Okamura K, Shirakusa T. A new surgical technique for voice restoration after laryngopharyngoesophagectomy with a free ileocolic graft: preliminary report. Surgery 1992;111(05):569-575

11 Chen HC, Gharb BB, Rampazzo A, Perrone F, Chen SH, Trignano E. Simultaneous restoration of voice function and digestive tract continuity in patients with synchronous primaries of hypopharynx and thoracic esophagus with pedicled ileocolon flap. Surgery 2011;149(05):662-671

12 Chen HC, Mardini S, Yang CW. Voice reconstruction using the free ileocolon flap versus the pneumatic artificial larynx: a comparison of patients' preference and experience following laryngectomy. J Plast Reconstr Aesthet Surg 2006;59(12):1269-1275

13 Karri V, Yang MC, Chung KP, Chen SH, Mardini S, Chen HC. Total pharyngolaryngectomy and voice reconstruction with ileocolon free flap: functional outcome and quality of life. J Plast Reconstr Aesthet Surg 2011;64(07):911-920

14 Carlson GW, Schusterman MA, Guillamondegui OM. Total reconstruction of the hypopharynx and cervical esophagus: a 20-year experience. Ann Plast Surg 1992;29(05):408-412
15 Nakatsuka T, Harii K, Asato H, et al. Analytic review of 2372 free flap transfers for head and neck reconstruction following cancer resection. J Reconstr Microsurg 2003;19(06):363-368, discussion 369

16 Grenz A, Clambey E, Eltzschig HK. Hypoxia signaling during intestinal ischemia and inflammation. Curr Opin Crit Care 2012;18(02):178-185

17 Eltzschig HK, Rivera-Nieves J, Colgan SP. Targeting the A2B adenosine receptor during gastrointestinal ischemia and inflammation. Expert Opin Ther Targets 2009;13(11):1267-1277

18 Chen HC, Patel H, Chen YC, Tang YB, Tan BK, Aydin A. Talking jejunum: a new, safe technique for voice reconstruction using free-jejunum transfer. Plast Reconstr Surg 2003;111(01): 336-340

19 Mardini S, Salgado CJ, Kim Evans KF, Chen HC. Reconstruction of the esophagus and voice. Plast Reconstr Surg 2010;126(02): 471-485

20 Rampazzo A, Gharb BB, Spanio di Spilimbergo S, Chung KP, Chen HC. Voice reconstruction with free ileocolon flap transfer: implications for the lower respiratory tract. Plast Reconstr Surg 2011;127(05):1916-1924

21 Saeki I, Matsuura T, Hayashida M, Taguchi T. Ischemic preconditioning and remote ischemic preconditioning have protective effect against cold ischemia-reperfusion injury of rat small intestine. Pediatr Surg Int 2011;27(08):857-862

22 Graeber GM, Wukich DK, Cafferty PJ, et al. Changes in peripheral serum creatine phosphokinase (CPK) and lactic dehydrogenase (LDH) in acute experimental colonic infarction. Ann Surg 1981; 194(06):708-715

23 Phillips L, Toledo AH, Lopez-Neblina F, Anaya-Prado R, ToledoPereyra LH. Nitric oxide mechanism of protection in ischemia and reperfusion injury. J Invest Surg 2009;22(01):46-55

24 Takada K, Yamashita K, Sakurai-Yamashita Y, et al. Participation of nitric oxide in the mucosal injury of rat intestine induced by ischemia-reperfusion. J Pharmacol Exp Ther 1998;287(01): 403-407

25 Asahara T, Murohara T, Sullivan A, et al. Isolation of putative progenitor endothelial cells for angiogenesis. Science 1997;275 (5302):964-967

26 Stellos K, Langer H, Gnerlich S, et al. Junctional adhesion molecule A expressed on human CD34+ cells promotes adhesion on vascular wall and differentiation into endothelial progenitor cells. Arterioscler Thromb Vasc Biol 2010;30(06):1127-1136

27 Chamoto K, Gibney BC, Lee GS, et al. CD34+ progenitor to endothelial cell transition in post-pneumonectomy angiogenesis. Am J Respir Cell Mol Biol 2012;46(03):283-289

28 Tanaka T, Narazaki M, Kishimoto T. IL-6 in inflammation, immunity, and disease. Cold Spring Harb Perspect Biol 2014;6(10): a016295 